\title{
REVIEW
}

\section{Emerging Approaches to the Surgical Management of Acute Traumatic Spinal Cord Injury}

\author{
Jefferson R. Wilson ${ }^{1}$ and Michael G. Fehlings ${ }^{1,2}$ \\ ${ }^{1}$ Division of Neurosurgery and Spinal Program, University of Toronto, Toronto, Ontario M5G 2C4, Canada; and ${ }^{2}$ University of \\ Toronto, Krembil Neuroscience Center, 399 Bathurst St, Toronto Western Hospital, Toronto, Ontario M5G 2C4, Canada
}

\begin{abstract}
Summary: Traumatic, spinal cord injury (SCI) is a potentially catastrophic event causing major impact at both a personal and societal level. To date, virtually all therapies that have shown promise at the preclinical stage of study have failed to translate into clinically effective treatments. Surgery is performed in the setting of SCI, with the goals of decompressing the spinal cord and restoring spinal stability. Although a consensus regarding the optimal timing of surgical decompression for SCI has not been reached, much of the preclinical and clinical evidence, as well as a recent
\end{abstract}

international survey of spine surgeons, support performing early surgery ( $<24$ hours). Results of the multicenter, Surgical Trial in Acute Spinal Cord Injury Study (STASCIS), expected later this year, should further clarify this important management issue. The overall goal of this review is to provide an update regarding the current status of surgical therapy for traumatic SCI by reviewing relevant pathophysiology, laboratory, and clinical evidence, as well as to introduce radiologic and clinical tools that aid in the surgical decision-making process. Key Words: Spinal cord injury, treatment, surgery, review.

\section{INTRODUCTION}

Traumatic spinal cord injury (SCI) is a devastating and debilitating condition that affects all regions of the world. In Canada, the annual incidence of SCI among adults is 42 per million and is diagnosed in approximately $5 \%$ of all major trauma victims $[1,2]$. Increasing life expectancy of spinal cord injured patients has lead to an increased worldwide prevalence of SCI, which is now approaching 2 million [3]. Economic data from the United States National Institute of Neurological Disorders and Stroke reports that approximately $\$ 4$ billion dollars are spent annually in the acute treatment and chronic care of spinal cord injured individuals [4]. In spite of the immense impact of SCI at a personal and societal level, uniform treatment strategies aimed at attenuating the initial degree of neurologic injury and reducing patients' future dependency are lacking [5].

Electronic supplementary material The online version of this article (doi:10.1007/s13311-011-0027-3) contains supplementary material, which is available to authorized users.

Address correspondence and reprint requests to: Michael G. Fehlings, M.D., Ph.D., FRCSC, FACS, University of Toronto, Krembil Neuroscience Center, 399 Bathurst St, Toronto Western Hospital, Toronto, ON, Canada. E-mail: michael.fehlings@uhn.on.ca.
Numerous acute therapies for SCI have appeared promising at the preclinical stages of study; however, most of these have failed to translate into clinically effective treatments [6]. From a pharmaceutical standpoint, all of the drug therapies tested in clinical efficacy trials have failed to demonstrate substantive benefit, with only high-dose methylprednisolone succinate showing modest success in the landmark National Acute Spinal Cord Injury Study (NASCIS)-2 trial [7]. However, due to the potential increased risk of complications with highdose methylprednisolone succinate, some clinicians have expressed concerns regarding this neuroprotective strategy $[8,9]$. The Sygen study was a phase III Randomized Control Trial (RCT) evaluating GM-1, a ganglioside complex with purported neuroprotective effects gleamed from preclinical studies [10]. Although the drug showed promise in improving neurologic recovery at a 3-month interim analysis, it ultimately failed to demonstrate efficacy with respect to the primary outcome at 6-months follow-up. Apart from these, numerous other pharmacologic agents, as well as cellular neuroregenerative therapies are under investigation for the treatment of acute SCI. Importantly, the preclinical models used to develop neuroprotective treatments all involve decompression of the cord prior to institution of the treatment, and it is noteworthy that 
surgical decompression was not universally applied in a standardized fashion on the NASCIS and Sygen studies.

Surgical therapy plays an important role in the care of spine trauma patients whether or not concomitant SCI exists. In the setting of SCI, surgery is performed with 2 interrelated goals: 1) to decompress the spinal cord in patients with neurologic deficit and ongoing spinal cord compression, and 2) realign the spinal column and restore spinal stability to facilitate early patient mobilization and eventual rehabilitation. In contrast to pharmaceutical therapies for SCI, in which several large well-designed clinical trials have been completed, there has been only 1 published prospective randomized control trial investigating surgical management of SCI [11]. The results of a recently completed prospective, multicenter trial (STASCIS) are expected this year and should help to better define the role of surgical management in acute traumatic SCI [12].

The overall goal of this review is to provide an update regarding the current status of surgical therapy for traumatic SCI by reviewing relevant pathophysiology, preclinical evidence, and existing and emerging clinical evidence, as well as to introduce radiological and clinical tools that aid in the surgical decision making process.

\section{PATHOPHYSIOLOGY}

The currently accepted model of SCI pathology involves a 2-hit hypothesis with both primary and secondary injury mechanisms leading to neural tissue destruction [13-15]. The primary injury consists of the initial traumatic compressive force applied to the spinal cord causing laceration and or intramedullary hematoma formation [14]. The compressive force is typically caused by bone or disc material that enters the spinal canal as a consequence of vertebral fracture or dislocation. Although primary preventive efforts aim to reduce the incidence of primary injuries, little can be performed from a therapeutic standpoint to repair this component of the injury once it has occurred. As a result of the initial mechanical event and persistent compression of the spinal cord, a cascade of secondary injury mechanisms are initiated that exacerbate the degree of tissue destruction. These processes, beginning immediately after the primary injury, include free radical formation, cellular ionic imbalance, cell membrane lipid peroxidation, release of excitotoxic glutamate, as well as vascular phenomenon, such as vasospasm and perfusion reperfusion injury [16-19]. The end result is the gradual expansion of the initial lesion, in a rostro-caudal direction from the epicenter of the initial force, furthering gray matter loss and white matter degeneration, at the expense of neurologic function [20]. Because the temporal evolution of secondary injury unfolds for a duration of several weeks, a therapeutic time window exists, during which mitigation of the aforementioned processes can lead to reduced neural tissue destruction and improved clinical outcomes.

\section{PRECLINICAL EVIDENCE FOR SURGICAL DECOMPRESSION}

Based on existing preclinical studies, it is clear that ongoing compression of the spinal cord represents a form of secondary injury that can be attenuated by decompressive surgery [21-31]. It is also evident that the degree of neural injury is directly related to the duration of spinal cord compression and inversely related to the time elapsed from injury to surgical decompression. Support for this relationship was demonstrated by Dimar et al. [24], when rats underwent weight drop induced SCI followed by persistent spinal cord compression of variable durations (i.e., at $0,2,6,24$, and 72 hours). The results indicated that for the 6-week recovery period the motor scores were consistently better for the rats with shorter duration of spinal cord compression. Additionally, portmortem histological analysis showed more severe neural tissue damage as the time until decompression increased. Summarizing the relevant literature on this topic, Furlan et al. [32] recently completed a systematic review of the available preclinical studies that have evaluated the role of surgical decompression in animal models of SCI with persistent cord compression [32]. Of the 19 included studies, 11 indicated a time-dependent effect of spinal cord compression in behavioral recovery, spinal cord blood flow, electrophysiological recovery, and extent of histopathological lesion. Based on the results of these and other studies, there is compelling biologic rationale to offer surgery in the case of traumatic SCI with persistent spinal cord compression. Furthermore, it seems that the strength of the neuroprotective effect seen with decompression is time dependent, with waning effectiveness as the duration of compression increases. Unfortunately, given the heterogeneity of these animal study models, it is difficult to extrapolate to clinical practice, a single efficacy cutoff time beyond which decompression becomes ineffective.

\section{CLINICAL EVIDENCE FOR SURGICAL DECOMPRESSION}

One of the largest challenges in translating the promising results of spinal cord decompression in animal studies into clinical practice has been to define a therapeutic time window during which performing decompression results in improved clinical outcomes. As for any therapy with purported neuroprotective effects, the earlier in the disease course therapy is instituted, the greater the potential for tissue preservation. Balanced with this biologic rationale for expedient 
therapy are the practical realities of trauma medicine, which in the real world setting, pose barriers to performing surgery in a timely fashion. In a feasibility study of surgical timing, the Toronto team (including the senior author) demonstrated that of those patients with traumatic SCI who underwent surgery, $24 \%$ of individuals had surgery by 24 hours and $40 \%$ of individuals had surgery by 48 hours [33]. In a similar European study, 51\% of patients received surgery prior to 24 hours [34]. Factors that can potentially increase the elapsed time from injury to surgery include pre-hospital transport time, time for diagnostic evaluation, and time for medical stabilization.

Taking these points into consideration, much attention has been paid in the literature to defining an ideal cutoff time, at which surgery provides neuroprotection and improved clinical outcomes within the practical time constraints of the real world. From this perspective, the 2 most intensively investigated cutoff points are 24 and 72 hours from the time of SCI [35]. Of studies using the 24$\mathrm{h}$ cutoff, the majority of the evidence is of low quality [32]. One systematic review, considered class 2 evidence, concluded that early surgery $(<24 \mathrm{~h})$ results in better neurological outcome than delayed surgery $(>24 \mathrm{~h})$ for patients with incomplete injuries [36]. In studies of lower methodological quality, surgery prior to $24 \mathrm{~h}$ has also been shown to improve neurological outcomes and to reduce complications, as well as certain measures of resource use, such as overall length of hospital stay and length of intensive care unit stay [37-39]. Conversely, studies of similar low methodological quality, using the same time cutoff, have failed to demonstrate a positive effect with respect to neurologic outcome [40, 41]. In examining studies using a 72-h cutoff to define early $v s$ late surgery, a similar dichotomy in outcomes exists. In the only randomized controlled trial on this topic to date, Vaccaro et al. [11] randomized patients to either early surgery $(<72 \mathrm{~h})$ or late surgery $(>72 \mathrm{~h})$, and found no difference in neurologic recovery or length of hospital stay between these groups. As a result of the large number of patients in this study that were lost to follow-up (20 of the original 62), this study is considered to provide class 2 evidence. In contrast to the findings of Vaccaro et al. [11], several other nonrandomized surgical series have demonstrated improved neurologic recovery and survival, as well as decreased length of hospital stay, when decompressive surgery was performed prior to 72 hours after SCI $[42,43]$.

Although there is no clear consensus from the older clinical literature regarding the optimal timing of surgery post-SCI, it is evident that early decompression is not associated with harm or increased complication rates. Furthermore, those clinical studies, which have found improvement in neurological recovery with early decompression, are supported by numerous preclinical studies demonstrating the time-dependent neuroprotective effects of decompressive surgery.

\section{International consensus and emerging evidence}

Interestingly, in spite of a clear answer on the topic of surgical timing for traumatic SCI, it is evident that the spine surgery community has embraced the concept of early surgery. As an addendum to the systematic review completed by the senior author's team, through the use of a modified Delphi process, a panel of 10 experts recommended that "surgical decompression of the injured spinal cord be performed within 24 hours when medically feasible" [32]. Furthermore, regarding this point, Fehlings et al. [44] performed a survey study in which orthopedic and neurosurgical spine surgeons were asked case-based questions regarding the optimal surgical management of traumatic SCI, with evidence of ongoing spinal cord compression. Of the 971 respondents, approximately $80 \%$ preferred to decompress the spinal cord within $24 \mathrm{~h}$ of injury, within every clinical scenario, except for central cord syndrome. In the case of an incomplete SCI, $72.9 \%$ of respondents would prefer to perform decompressive surgery within $6 \mathrm{~h}$. These results implicate that although we lack a final answer regarding the relative efficacy of early surgery, a large proportion of the international spine community consider it a priority based on the available preclinical and clinical evidence combined with their own personal experience.

To provide a definitive answer regarding the optimal timing of decompressive surgery for SCI, a large scale, multicenter, prospective trial, STASCIS, is underway. The primary study objective is to evaluate the effects of early ( $<24 \mathrm{~h}$ after injury) $v s$ late ( $\geq 24 \mathrm{~h}$ after injury) decompressive surgery on neurologic recovery after cervical SCI. As secondary questions, the impact of surgical timing on rates of acute inpatient complications, as well as overall survival, will also be considered. With patient enrollment finishing in 2009, complete study results are anticipated in the coming year, with preliminary data ostensibly supportive of the hypothesis that decompression before $24 \mathrm{~h}$ results in improved neurologic recovery [12].

\section{SURGICAL PATIENT SELECTION AND OPERATIVE APPROACH}

\section{Radiologic criteria}

The potential benefit of surgical decompression in the setting of traumatic SCI is contingent on the existence of ongoing spinal cord compression. Hence, having access to a reliable quantitative method for radiographically assessing the degree of spinal canal narrowing and spinal cord compression is critical when determining patients' potential for benefit from surgical intervention. Conventional X-ray, computed tomography (CT) and magnetic resonance imaging (MRI) have all been evaluated for their usefulness in this regard. To determine spinal canal compromise measures, such as anteroposterior (AP) canal diameter, transverse canal diameter, canal area, and the ratio 
of AP diameter to transverse diameter have been used [45-49]. Similarly, to quantify spinal cord compression, measures examined to date include AP cord diameter, transverse cord diameter, cord area, cord circumference, and cord circularity [50-53]. Unfortunately the use of these measures are limited because there are few assessments of their individual psychometric properties or values for their sensitivity and specificity [54].

To provide a reliable means to interpret imaging in the context of acute SCI, Fehlings et al. [55] performed a multicenter study defining the optimal radiologic method for assessing spinal cord compression and spinal canal compromise [55]. In this study, 2 radiologists evaluated the acute admission imaging (X-Ray, CT, and MRI) of 71 patients with cervical SCI. For purposes of standardization, maximal spinal canal compromise was calculated by comparing the AP canal diameter at the level of maximum injury with the AP canal diameter at nearest normal levels above and below (Table 1). Similarly, maximal spinal cord compression was calculated by comparing the AP cord diameter at the level of maximum injury with the AP cord diameter at nearest normal levels above and below (Table 1). Using these methods, sagittal CT and T1-weighted MRI were most effective at evaluating spinal canal compromise, whereas sagittal T2-weighted MRI was most effective at evaluating spinal cord compression. Interobserver correlations of measurements between study radiologists were consistently strong and statistically significant. Interestingly, the presence of $>25 \%$ canal compromise on sagittal CT predicted the MRI presence of cord compression in $100 \%$ of cases; however, in those with $<25 \%$ canal compromise on $\mathrm{CT}$, the majority continued to exhibit MRI evidence of cord compression. This finding illustrates the high sensitivity, but very poor specificity of canal compromise visualized on CT in predicting spinal cord compression. Overall, this study has provided a standardized and reliable method for quantifying the degree of canal compromise and cord compression, to be used as a

Table 1. Standardized Formulas for Determining Maximal Canal Compromise And Maximal Cord Compression.

\begin{tabular}{cc}
\hline Maximal Canal & Maximal Cord \\
Compromise (\%) & Compression (\%) \\
\hline$((1-\mathrm{Di}) /(\mathrm{Da}+\mathrm{Db} / 2)) \times 100 \%$ & $((1-\mathrm{di}) /(\mathrm{da}+\mathrm{db} / 2)) \times 100 \%$ \\
\hline
\end{tabular}

Modified from: Fehlings MG, Rao SC, Tator CH, et al. The optimal radiologic method for assessing spinal canal compromise and cord compression in patients with cervical spinal cord injury part 2: results of a multicenter study. Spine 1999;24:605-613.

$D i=$ anteroposterior (AP) canal diameter at the level of maximal injury; $\mathrm{Da}=\mathrm{AP}$ canal diameter at nearest normal level above level of injury; $\mathrm{Db}=\mathrm{AP}$ diameter at nearest normal level below level of injury; $\mathrm{di}=\mathrm{AP}$ diameter of the cord at the level of maximal injury; $\mathrm{da}=\mathrm{AP}$ diameter of cord at nearest normal level above level of injury; $\mathrm{db}=\mathrm{AP}$ diameter of the cord at nearest normal level below level of injury. benchmark in the clinical realm and for incorporation into future and current surgical studies in SCI.

\section{Clinical algorithms to aid in surgical decision-making}

The presence of spinal cord compression and neurologic deficit are important considerations when assessing if a patient with spinal trauma requires surgery. However, additional concepts, such as mechanism of injury, biomechanical stability, osteo-ligamentous integrity and fracture morphology also define the nature and severity of a given spine injury and help to guide therapeutic decision-making. Several authors have attempted to incorporate some or all of these elements, to create a comprehensive spinal trauma classification system, for use in the clinical realm. These have included the 2column model of Holdsworth [56], the 3-column model of Denis [57], the AO thoracolumbar injury classification and the McCormack load-sharing classification [5659]. Although each of these offers a unique profile of advantages and disadvantages, no single system has gained widespread use for several reasons. First, the majority of these are complex, nonintuitive, and cumbersome for use in an everyday clinical setting. Second, most systems fail to incorporate modern day diagnostic imaging modalities, such as MRI, which are capable of assessing the integrity of soft-tissue structures critical for maintaining spinal stability. Such structures include the posterior ligamentous complex in the thoracolumbar spine and disco-ligamentous complex in the cervical spine. Third, most systems fail to guide clinical treatment decisions based on specific injury characteristics. Of particular interest would be the development of objective standardized criteria, which would dictate the need for operative $v s$ conservative management for a specific case. Finally, few systems consider SCI or the pattern of neurologic deficit in their classification, features which obviously impact the need for and urgency of surgical treatment.

To overcome the recognized deficiencies of the existing systems, members of the Spine Trauma Study Group (STSG) have recently participated in the development of 2 novel classification systems for spinal trauma [60, 61]. The STSG is a working group dedicated to the study of traumatic conditions of the human spine, consisting of 50 surgeons from 12 countries around the world. The first classification system, known as the Subaxial Injury Classification (SLIC), addresses injuries to the subaxial cervical spine [60]. Based on a review of the literature and survey of the STSG expert committee, clinical and radiographic variables were identified as candidates for incorporation into SLIC. Ultimately, the classification system consists of 3 main categories: 1) injury/fracture morphology, 2) integrity of disco-ligamentous complex, and 3) neurological status (Table 2). Injury morphology is subdivided into: 1) compression injuries, 2) distraction 
Table 2. The Subaxial Cervical Spine Injury Classification System.

\begin{tabular}{lc}
\hline \multicolumn{1}{c}{ Injury Variable } & $\begin{array}{c}\text { Weighted } \\
\text { Severity Points }\end{array}$ \\
\hline Morphology: & \\
No abnormality & 0 \\
Compression & 1 \\
Distraction & 3 \\
Rotation/Translation & 4 \\
Disco-ligamentous complex integrity: & \\
Intact & 0 \\
Indeterminate & 1 \\
Disrupted & 2 \\
Neurologic status: & \\
Intact & 0 \\
Root injury & 1 \\
Complete cord injury & 2 \\
Incomplete cord injury & 3 \\
Cord Compression with neurodeficit & +1 \\
\hline
\end{tabular}

Modified from: Vaccaro AR, Hurlbert J, Patel AA, et al. The subaxial cervical spine injury classification system: a novel approach to recognize the importance of morphology, neurology, and inegrity of the disco-ligamentous complex. Spine 2007;32:2365-2374.

injuries, and 3) translation/rotation injuries. Integrity of disco-ligamentous complex is assessed to be either 1) intact, 2) indeterminate (interspinous spreading, or soft tissue T2 hyperintensity), or 3) disrupted (facet dislocation or disc space widening). Neurological status is classified as: 1) intact, 2) radiculopathy, 3) incomplete SCI, or 4) complete $\mathrm{SCI}$. Within the 3 categories, each variable is assigned a weighted score depending on how it contributes to the overall injury severity. For a given injury, summing the weighted scores across each category leads to an overall injury severity score (SLIC score). The authors have recommended that patients with a SLIC score of less than 4 should be treated nonoperatively, those with an SLIC score greater than 4 should be treated operatively, and those with an SLIC score of exactly 4 can be managed with or without surgery at the discretion of the clinical management team. The reliability of the overall SLIC classification is substantial with intra-rater intraclass correlation and interrater intraclass correlation measurements of 0.83 and 0.71 , respectively.

The second classification system contributed by the STSG, known as the Thoracolumbar Injury Classification and Severity Score (TLICS), addresses spinal injuries at the thoracolumbar junction based on an algorithm virtually identical to SLIC (Table 3) [61]. As with the SLIC, the TLICS evaluates injuries on 3 categories, each of which have weighted subdivisions that contribute to an overall severity score. Study authors provided the recommendation that injuries evaluated to have a TLICS score less than 4 should be managed nonoperatively, those with a score greater than 4 should be managed operatively, and those with a score of exactly 4 might be managed with or without surgery. Similar to the SLIC, TLICS has shown good to excellent inter-rater and intra-rater reliability in assessment of its psychometric properties.

These classifications have provided a standardized, reproducible and reliable means of classifying cervical and thoracolumbar trauma. Moreover, they provide clinicians with a systematic way of determining the optimal treatment strategy for a given patient based on individual injury characteristics.

\section{Operative approach}

When the combination of clinical and radiologic information for a given patient with SCI dictate the need for surgical therapy, many questions remain for the surgeon involved. These decision points include choice of operative approach (anterior vs posterior $v s$ combined), number of levels to be included in decompression or fusion, use of bone graft (autograft, allograft, synthetic), use of instrumentation, use of supplements, such as bone cement or bone morphogenic protein, and use of intraoperative aids, such as neurophysiology or neuronavigation. Regarding the operative approach, conventional wisdom dictates that a decision to approach anteriorly or posteriorly should be based on the location of the primary pathology. Hence, in the case of a cervical spinal cord injury with a large protruding disc compressing the spinal cord from the anterior, the choice to decompress and fuse from an anteriorly directed surgical approach is intuitive. Conversely, in treating some injuries, there appears to be equipoise in the choice of approach, with neither anterior nor posterior seeming intuitively superior. To address such injuries, Brodke et al. [62] randomized 52 patients with subaxial cervical spine injuries, with concomitant SCI, to either anterior or posterior stabilization and fusion. Patients requiring a

Table 3. The Thoracolumbar Injury Classification and Severity Score.

\begin{tabular}{ll}
\hline \multicolumn{1}{c}{ Injury Variable } & $\begin{array}{c}\text { Weighted } \\
\text { Severity Points }\end{array}$ \\
\hline Morphology: & 0 \\
No abnormality & 1 \\
Compression & 3 \\
Translation/Rotation & 4 \\
Distraction & \\
Posterior Ligamentous Complex integrity: & 0 \\
Intact & 2 \\
Indeterminate & 3 \\
Disrupted & \\
Neurologic status: & 0 \\
Intact & 2 \\
Root injury & \\
Conus, cord injury: & 3 \\
Incomplete cord injury & 2 \\
Complete & 3 \\
Cauda equina & \\
\hline
\end{tabular}

Modified from: Vaccaro AR, Lehman RA, Hurlbert RJ, et al. A new classification of thoracolumbar injuries: the importance of injury morphology, the integrity of the posterior ligamentous complex, and neurologic status. Spine 2005;30:2325-2333. 


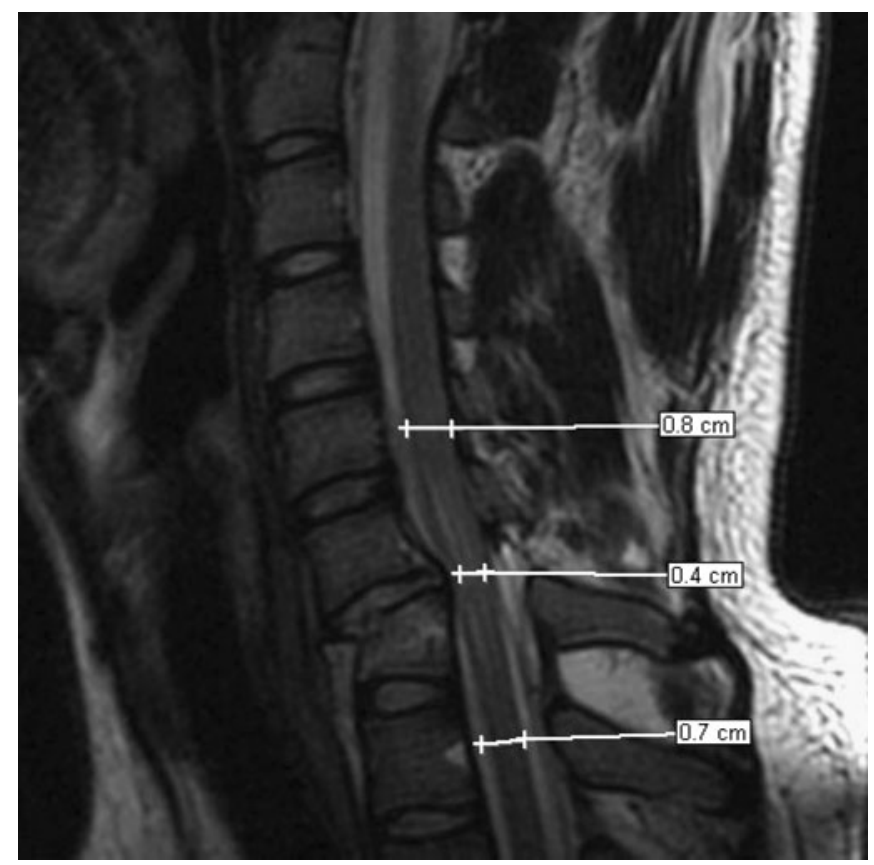

FIG. 1. Preoperative T2-weighted cervical spinal magnetic resonance image demonstrating spinal cord compression at level C6-C7 with concomitant hyperintense signal changes within the cord. Based on the formula from Table 1, the degree of spinal cord compression is (1 $[0.4 /(0.8+0.7) / 2]) \times 100 \%=53 \%$.

specific approach for decompression or reduction were excluded. Between the two approaches, there were no significant differences in spinal fusion rates, alignment, neurologic recovery, or long-term complications.

Practically, for questions of approach or other operative related issues, it is up to the surgeon to combine the best available evidence with their own anecdotal experience to create a treatment plan tailored to the patient and the specifics of the clinical scenario.

\section{Illustrative clinical case}

A 20-year-old male driver was involved in a motor vehicle rollover accident while unrestrained. His neurological examination on arrival at hospital demonstrated

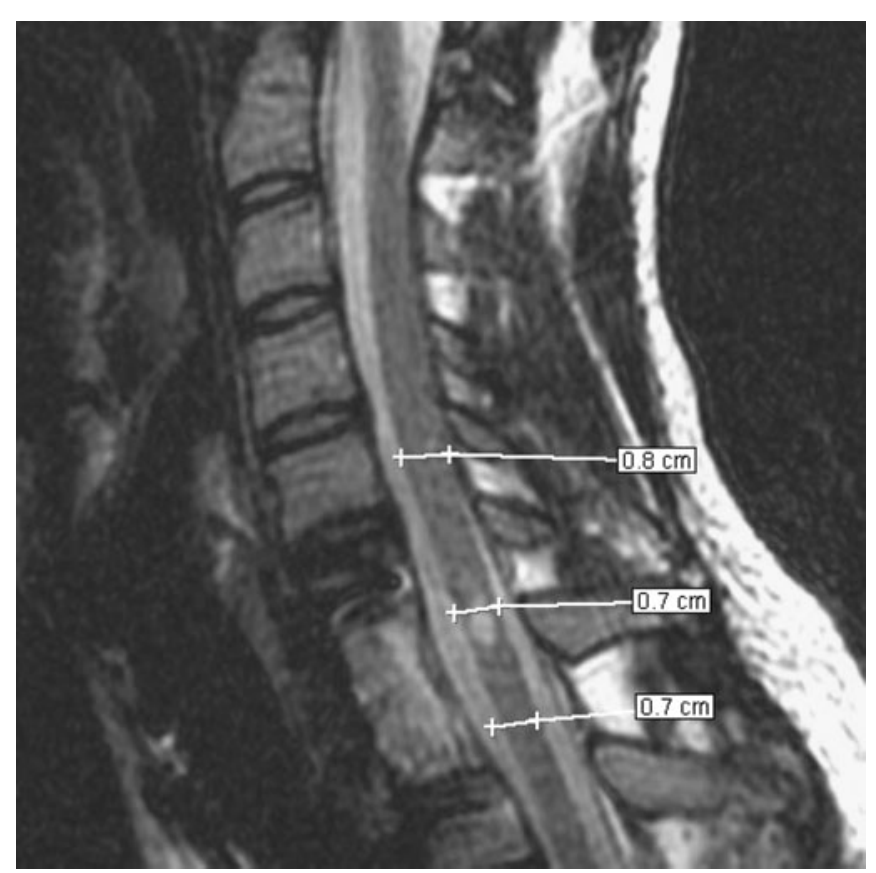

FIG. 2. Postoperative T2-weighted cervical spinal magnetic resonance image at 1-year postinjury demonstrating complete decompression of the spinal cord and restoration of normal cervical spinal alignment. 
an incomplete, ASIA C SCI with a neurologic level of injury at $\mathrm{C} 7$. A cervical $\mathrm{CT}$ scan with 3-dimensional reconstructions was performed showing a flexion/distraction injury with facet dislocation and anterior translation of C6 vertebral body with respect to C7. An MRI scan of the cervical spine demonstrated spinal cord compression at the C6-C7 level with associated T2 hyperintensity in the spinal cord at this level. FIG. 1 illustrates the degree of spinal cord compression (53\%) on the preoperative MRI, as measured by the standardized method just previously described. Given the ongoing spinal cord compression and a calculated SLIC score of 10 (morphology +4 , disco-ligamentous complex +2 , neurologic status +3 , cord compression +1 ), the patient underwent surgical management after closed reduction was achieved $12 \mathrm{~h}$ after the injury. A C7 corpectomy with autologous bone graft placement followed by a posterior lateral mass fixation was performed. At 1-year postinjury, the patient had improved to an ASIA D injury severity status with follow-up MRI demonstrating normal cervical alignment with no evidence of spinal cord compression or spinal canal compromise (FIG. 2).

\section{CONCLUSIONS}

With descriptions appearing in ancient Egyptian medical texts more than 4,000 years old, SCI, as a clinical entity, has perplexed clinicians and scientists alike for centuries. In spite of the large volume of basic science and clinical research that has accumulated on this topic to date, a major therapeutic breakthrough has remained elusive. Although far from a cure, the preclinical and existing clinical evidence evaluating early surgical decompression for traumatic spinal cord injury appears promising. We look forward to providing a definitive answer on this topic once the STASCIS data has been fully analyzed. Ultimately, we believe that the solution to optimizing clinical outcomes in SCI is complex and will involve a combinatorial approach that may include surgery, in addition to other neuroprotective and cellular regenerative therapies. Based on a critical analysis of the best available data, in concert with surveys of current surgical opinion, we strongly recommend the early application of surgical decompression and spinal stabilization within $24 \mathrm{~h}$ of SCI when medically feasible.

Acknowledgments: Full conflict of interest disclosure is available in the electronic supplementary material for this article.

\section{REFERENCES}

1. Ackery A, Tator C, Krassioukov A. A global perspective on spinal cord injury epidemiology. J Neurotrauma 2004;21:1355-1370.
2. Pirouzmand F. Epidemiological trends of spine and spinal cord injuries in the largest Canadian adult trauma center from 1986 to 2006. J Neurosurg Spine 2010:12:131-140.

3. Wyndaele M, Wyndaele JJ. Incidence, prevalence and epidemiology of spinal cord injury: what learns a worldwide literature survey? Spinal Cord 2006;44:523-529.

4. Baptiste DC, Fehlings MG. Emerging Drugs for Spinal Cord Injury. Expert Opinion on Emerging Drugs 2008;13:63-80.

5. Hawryluk GW, Rowland J, Kwon BK, et al. Protection and repair of the injured spinal cord: a review of completed, ongoing, and planned clinical trials for acute spinal cord injury. Neurosurg Focus 2008;25:E14.

6. Rowland JW, Hawryluk GW, Kwon B, et al. Current status of acute spinal cord injury pathophysiology and emerging therapies: promise on the horizon. Neurosurg Focus 2008;25:E2.

7. Braken MB, Shepard MJ, Collins WF, et al. A randomized, controlled trial of methylprednisolone or naloxone in the treatment of acute spinal-cord injury. Results of the Second National Acute Spinal Cord Injury Study. N Engl J Med 1990;322:1405-1411.

8. Bracken MB, Shepard MJ, Holford TR, et al. Administration of methylprednisolone for 24 or 48 hours or tirilazad mesylate for 48 hours in the treatment of acute spinal cord injury. Results of the Third National Acute Spinal Cord Injury Randomized Controlled Trial. National Acute Spinal Cord Injury Study. JAMA 1997;277:1597-1604.

9. Hurlbert RJ. Methylprednisolone for acute spinal cord injury: an inappropriate standard of care. J Neurosurg (Spine) 2000;93:1-7.

10. Geisler FH, Coleman WP, Grieco G, et al. The Sygen multicenter acute spinal cord injury study. Spine 2001;26:S87-S98.

11. Vaccaro AR, Daugherty RJ, Sheehan TP, et al. Neurologic outcome of early versus late surgery for cervical spinal cord injury. Spine 1997;22:2609-2613

12. Fehlings MG, Arvin B. The timing of surgery in patients with central spinal cord injury. J Neurosurg (Spine) 2009;10:1-2.

13. Fehlings MG, Sekhon L. Celluslar, ionic and biomolecular mechanisms of the injury process. In: Benzel, Tator $\mathrm{CH}$, eds. Contemporary Management of Spinal Cord Injury: From Impact to Rehabilitation. Chicago, IL: American Association of Neurologic Surgeons, 2000:33-50.

14. Tator $\mathrm{CH}$, Fehlings MG. Review of the secondary injury theory of acute spinal cord trauma with emphasis on vascular mechanisms. J Neurosurg 1991;75:15-26.

15. Amar AP, Levy ML. Pathogenesis and pharmacological strategies for mitigating secondary damage in acute spinal cord injury. Neurosurgery 1999;44:1027-1039.

16. Braughler JM, Duncan LA, Chase RL. Interaction of lipid peroxidation and calcium in the pathogenesis of neuronal injury. Cent Nerv Syst Trauma 1985;2:269-283.

17. Jorgensen MB, Diemer NH. Selective neuron loss after cerebral ischemia in the rat: possible role of transmitter glutamate. Acta Neurol Scand 1982;66:536-546.

18. Rothman SM, Olney JW. Glutamate and the pathophysiology of hypoxic-ischemic brain damage. Ann Neurol 1986;19:105-111.

19. Wagner FC, Stewart WB. Effect of trauma dose on spinal cord edema. J Neurosurg 1981;54:802-806.

20. Schwartz G, Fehlings MG. Secondary injury mechanisms of spinal cord trauma: a novel therapeutic approach for the management of secondary pathophysiology with the sodium channel blocker riluzole. Prog Brain Res 2002;137:177-190.

21. Brodkey JS, Richards DE, Blasingame JP, et al. Reversible spinal cord trauma in cats. Additive effects of direct pressure and ischemia: J Neurosurg 1972 Nov;37(5):591-593.

22. Carlson GD, Minato Y, Okada A et al. Early time-dependent decompression for spinal cord injury: Vascular mechanisms of recovery. J Neurotrauma 1997;14:951-962.

23. Delamarter RB, Sherman J, Carr JB. Pathophysiology of spinal cord injury. Recovery after immediate and delayed decompression. J Bone Joint Surg Am 1995;77:1042-1049.

24. Dimar JR II, Glassman SD, Raque GH, et al. The influence of spinal canal narrowing and timing of decompression on neurologic recovery after spinal cord contusion in a rat model. Spine 1999;24:1623-1633.

25. Dolan EJ, Tator CH, Endrenyi L. The value of decompression for acute experimental spinal cord compression injury. J Neurosurg $1980 ; 53: 749-755$ 
26. Guha A, Tator CH, Endrenyi L, et al. Decompression of the spinal cord improves recovery after acute experimental spinal cord compression injury. Paraplegia 1987;25:324-339.

27. Kobrine AI, Evans DE, Rizzoli HV. Experimental acute balloon compression of the spinal cord. Factors affecting disappearance and return of the spinal evoked response. J Neurosurg 1979;51:841-845.

28. Nystrom B, Berglund JE. Spinal cord restitution following compression injuries in rats. Acta Neurol Scand 1988;78:467-472.

29. Tarlov IM. Spinal cord compression studies. III. Time limits for recovery after gradual compression in dogs. AMA Arch Neurol Psychiatry 1954;71:588-597.

30. Tarlov IM, Klinger H. Spinal cord compression studies. II. Time limits for recovery after acute compression in dogs. AMA Arch Neurol Psychiatry 1954;71:271-290.

31. Carlson GD, Gorden CD, Oliff HS, et al. Sustained spinal cord compression: Part I: Time-dependent effect on long-term pathophysiology. J Bone Joint Surg Am 2003;85:86-94.

32. Furlan JC, Noonan V, Cadotte DW, et al. Timing of decompressive surgery of spinal cord after traumatic spinal cord injury: an evidence-based examination of pre-clinical and clinical studies. J Neurotrauma 2010;27:1-29.

33. Tator $\mathrm{CH}$, Fehlings MG, Thorpe K, et al. Current use and timing of spinal surgery for management of acute spinal cord injury in North America: results of a retrospective multicenter study. J Neurosurg 1999;91:150-154.

34. Botel U, Glaser E, Niedeggen A. The surgical treatment of acute spinal paralyzed patients. Spinal Cord 1997;35:420-428.

35. Fehlings MG, Perrin RG. The timing of surgical intervention in the treatment of spinal cord injury: a systematic review of recent clinical evidence. Spine 2006;31:S28-S35.

36. La Rosa G, Conti A, Cardali S, et al. Does early decompression improve neurological outcome of spinal cord injured patients? Appraisal of the literature using a meta-analytical approach. Spinal Cord 2004;42:503-512.

37. Levi I, Wolf A, Rigamonti D, et al. Anterior decompression in cervical spine trauma: does the timing of surgery affect the outcome? Neurosurgery 1991;29:216-222.

38. Campagnolo D, Esquieres RE, Kopacz KJ. Effect of timing of stabilization on length of stay and medical complication following spinal cord injury. J Spinal Cord Med 1997;20:331-334.

39. McLain RF, Benson DR. Urgent surgical stabilization of spinal fractures in polytrauma patients. Spine 1999;24:1646-1654.

40. Duh MS, Shepard MJ, Wilberger JE, et al. The effectiveness of surgery on the treatment of acute spinal cord injury and its relation to pharmacologic treatment. Neurosurgery 1994;35:240-248.

41. Pollard ME, Apple DF. Factors associated with improved neurologic outcomes in patients with incomplete tetraplegia. Spine 2003;28:33-39.

42. Clohisy JC, Akbarnia BA, Bucholz RD, et al. Neurologic recovery associated with anterior decompression of spine fractures at the thoracolumbar junction (T12-L1). Spine 1992;17:S325-S330.

43. Mirza SK, Krengel WF, Chapman JR, et al. Early versus delayed surgery for acute cervical spinal cord injury. Clin Orthop Relat Res 1999;359:104-114.

44. Fehlings MG, Rabin D, Sears W, et al. Current practice in the timing of surgical intervention in spinal cord injury. Spine 2010;35: S166-S173.
45. Denno JJ, Meadows GR. Early diagnosis of cervical spondylotic myelopathy: A useful clinical sign. Spine 1991;16:1353-1355.

46. Edwards WC, LaRocca H. The development segmental sagittal diameter of the cervical spinal canal in patients with cervical spondylosis. Spine 1983;8:20-27.

47. Eismont FJ, Clifford S, Goldberg M, et al. Cervical sagittal spinal canal size in spine injury. Spine 1984;9:663-666

48. Matsuura P, Waters RL, Adkins RH, et al. Comparison of computerized tomography parameters of the cervical spine in normal control subjects and spinal cord-injured patients. J Bone Joint Surg [Am] 1989;71:183-188.

49. Stanley JH, Schabel SI, Frey GD, Hungerford GD. Quantitative analysis of the cervical spinal canal by computed tomography. Neuroradiology 1986;28:139-143.

50. Fujiwara K, Yonenobu K, Hiroshima K, et al. Morphometry of the cervical spinal cord and its relation to pathology in cases with compression myelopathy. Spine 1988;13:1212-1216.

51. Hayashi $\mathrm{K}$, Yone $\mathrm{K}$, Ito $\mathrm{H}$, et al. MRI findings in patients with a cervical spinal cord injury who do not show radiographic evidence of a fracture or dislocation. Paraplegia 1995;33:212-215.

52. Matsuyama Y, Kawakami N, Mimatsu K. Spinal cord expansion after decompression in cervical myelopathy: investigation by computed tomography, myelography, and ultrasonography. Spine 1995;20:1657-1663

53. Yu YL, du Boulay GH, Stevens JM, et al. Computed tomography in cervical spondylotic myelopathy and radiculopathy: visualization of structures, myelographic comparison, cord measurements and clinical utility. Neuroradiology 1986;28:221-236.

54. Rao SC, Fehlings MG. The optimal radiologic method for assessing spinal canal compromise and cord compression in patients with cervical spinal cord injury part 1: an evidenced based analysis of the published literature. Spine 1999;24:598-604.

55. Fehlings $\mathrm{MG}$, Rao $\mathrm{SC}$, Tator $\mathrm{CH}$, et al. The optimal radiologic method for assessing spinal canal compromise and cord compression in patients with cervical spinal cord injury part 2: results of a multicenter study. Spine 1999;24:605-613.

56. Holdsworth FW. Fractures, dislocation and fracture-dislocation of the spine. JBJS 1963;45:6-20.

57. Denis F. The three column spine and its significance in the classification of acute thoracolumbar spinal injuries. Spine $1983 ; 8: 817-831$.

58. McCormack T, Karaikovic E, Gaines RW. The load sharing classification of spine fractures. Spine 1994;19:1741-1744.

59. Magerl F, Aebi M, Gertzbein SD, et al. A comprehensive classification of thoracic and lumbar injuries. Eur Spine J 1994;3:184-201.

60. Vaccaro AR, Hurlbert J, Patel AA, et al. The subaxial cervical spine injury classification system: a novel approach to recognize the importance of morphology, neurology, and inegrity of the discoligamentous complex. Spine 2007;32:2365-2374.

61. Vaccaro AR, Lehman RA, Hurlbert RJ, et al. A new classification of thoracolumbar injuries: the importance of injury morphology, the integrity of the posterior ligamentous complex, and neurologic status. Spine 2005;30:2325-2333.

62. Brodke DS, Anderson PA, Newell DW, et al. Comparison of anterior and posterior approaches in cervical spinal cord injuries. J Spin Disord Tech 2003;16:229-235. 